\title{
SCREENING EFFECTS ON NONRELATIVISTIC BREMSSTRAHLUNG IN THE SCATTERING OF ELECTRONS BY NEUTRAL ATOMS
}

\author{
YOUNG-DAE JUNG and KUN-SANG LEE
}





\title{
SCREENING EFFECTS ON NONRELATIVISTIC BREMSSTRAHLUNG IN THE SCATTERING OF ELECTRONS BY NEUTRAL ATOMS
}

\author{
YOUNG-DAE JUNG ${ }^{1}$ \\ Space Science Laboratory, NASA/Marshall Space Flight Center \\ AND \\ Kun-SANG LEE \\ Department of Physics, Hanyang University, Ansan, Kyunggi-Do 425-791, South Korea \\ Received 1994 July 12 ; accepted 1994 August 31
}

\begin{abstract}
Atomic screening effects on nonrelativistic electron-atom bremsstrahlung radiation are investigated using a simple analytic solution of the Thomas-Fermi model for many-electron atoms. The Born approximation is assumed for the initial and final states of the projectile electron. The results show that the screening effect is important in the soft radiation region and is decreasing with increasing radiation. These results help provide correct information about the behavior of bound electrons in the target atom in bremsstrahlung processes.

Subject headings: atomic processes - radiation mechanisms: nonthermal
\end{abstract}

\section{INTRODUCTION}

Electron bremsstrahlung radiation has received much attention because of its applications in many areas of physics, such as atomic physics, astrophysics, and plasma physics. However, most of the literature has dealt with electron-nucleus bremsstrahlung radiation rather than electron-atom bremsstrahlung. Although some authors (see Heitler 1954; Bethe \& Salpeter 1957) have already investigated the screening effects of the bound electrons in the target atom, their cases are extreme relativistic bremsstrahlung processes. Thus, in this paper, we investigate the screening effects on electron-atom bremsstrahlung radiation in the nonrelativistic limit because nonrelativistic bremsstrahlung processes are known to be an important source of continuum $\mathrm{X}$-ray radiation and because this situation is quite different from the extreme relativistic case. Also, it is well known that in astrophysical environments an important source of opacity is inverse bremsstrahlung in the scattering of electrons by neutral atoms. The screening effect of the atomic electrons plays an important role in the cross section itself, as well as in the radiation power spectrum. In this paper we use the nonrelativistic Born approximation for both the initial and the final states of the projectile election. The potential energy of a many-electron atom is given by an analytic solution of the Thomas-Fermi field suggested by Mott $\&$ Massey (1965). Our result is perhaps useful and can readily be applied to a number of associated problems in atomic physics, astrophysics, and plasma physics since we obtain a simple analytic solution in terms of the atomic parameters.

In $\S 2$ we derive the general formulation for the electron bremsstrahlung cross section using the nonrelativistic Born approximation. In $\S 3$ we obtain a simple analytic form of the electron-atom bremsstrahlung radiation cross section using an analytic solution of the Thomas-Fermi model for many-electron atomic systems. In $\$ 4$ a comparison is made between the electron-atom and electron-ion bremsstrahlung spectra. Finally, in $\S 5$ we discuss these results and their applications.

\section{NONRELATIVISTIC BREMSSTRAHLUNG CROSS SECTION}

\subsection{Formulations}

If we assume the target atom is at rest and make use of second-order nonrelativistic perturbation theory (see Gould 1990), the differential cross section for the electron-atom bremsstrahlung process is

$$
d^{2} \sigma_{b}=d \sigma_{\mathrm{C}} d W_{\omega}
$$

where $d \sigma_{\mathrm{C}}$ is the differential elastic Coulomb-scattering cross section,

$$
d \sigma_{\mathrm{C}}=\frac{1}{2 \pi h^{2} v_{0}^{2}}|\tilde{V}(q)|^{2} q d q,
$$

and $\tilde{V}(q)$ is the Fourier transform of the Coulomb potential $V(r)$,

$$
\tilde{V}(\boldsymbol{q})=\int d^{3} r e^{-i \boldsymbol{q} \cdot r} V(\boldsymbol{r}) .
$$

Here $\boldsymbol{q}\left(=\boldsymbol{k}_{0}-\boldsymbol{k}_{f}\right)$ is the momentum transfer and $\boldsymbol{k}_{0}$ and $\boldsymbol{k}_{f}$ are the wavevectors of the initial and final states of the projectile electron, respectively. And, $d W_{\omega}$ is the photon emission probability in the solid angle $d \Omega$ :

$$
d W_{\omega}=\frac{\alpha}{4 \pi^{2}} \Lambda^{2} \sum_{e}|e \cdot \boldsymbol{q}|^{2} \frac{d \omega}{\omega} d \Omega,
$$

' Current postal address: Department of Physics, Hanyang University, Ansan, Kyunggi-Do 425-791, South Korea. 

where $\alpha\left(=e^{2} / h c\right)$ and $\Lambda(=h / m c)$ are the fine-structure constant and the Compton wavenumber, respectively. After some algebra, we can define the differential bremsstrahlung radiation cross section as

$$
\frac{d^{2} \chi_{\mathrm{b}}}{d \omega d q}=\frac{d^{2} \sigma_{\mathrm{b}}}{d \omega d q} h \omega
$$

For a bare nucleus target with charge $Z$, the unscreened Coulomb potential is

$$
V_{n}(\boldsymbol{r})=-\frac{Z e^{2}}{r}
$$

In this case, the calculation of the cross section is straightforward and is known as the nonrelativistic Bethe-Heitler formula. This will be briefly discussed in the following subsection. For an atomic target system, in most cases the polarization of the atomic electrons by the incident electron is unimportant and the potential $V_{a}(r)$ is of form

$$
V_{a}(\boldsymbol{r})=-\frac{Z e^{2}}{r}+\sum_{j} \frac{e^{2}}{\left|\boldsymbol{r}-\boldsymbol{r}_{j}\right|},
$$

where $r_{j}$ is the position vector of the $j$ th bound electron. In this case, the calculation of the cross section is complicated by the electron-electron interaction term. A recent paper by Jung \& Gould (1991) gave prescriptions for screening constants and effective nuclear charges for bound electrons in many-electron atoms using the orthogonal Slater orbitals. These results are very useful for describing the total and individual electron states up to $3 d$ electrons. However, the total average potential is more convenient to evaluate for atomic processes involving many-electron systems, owing to its great simplicity. Thus, in this paper, we employ the Thomas-Fermi model for many-electron atoms.

\subsection{Nonrelativistic Bethe-Heitler Equation}

In this subsection we will briefly review the nonrelativistic Bethe-Heitler formulation for electron-pure-ion bremsstrahlung radiation. In this case the differential bremsstrahlung radiation cross section is readily obtained by the Fourier transform of the pure Coulomb potential (eq. [6]). Thus, $d \chi_{b} / d \omega$ for the electron-ion bremsstrahlung process is given by

$$
\left(\frac{d \chi_{b}}{d \omega}\right)_{e^{-}-Z e}=\frac{16}{3} \frac{Z^{2} e^{2}}{c}\left(\frac{e^{2}}{m c^{2}}\right)^{2} \frac{1}{\beta^{2}} \ln \left(\frac{\sqrt{\epsilon_{0}}+\sqrt{\epsilon_{0}-\epsilon}}{\sqrt{\epsilon_{0}}-\sqrt{\epsilon_{0}-\epsilon}}\right),
$$

where $\epsilon_{0}=E_{0} \mathrm{ryd}^{-1}$ and $\epsilon=\hbar \omega$ ryd $^{-1}$. Equation (8) is called the nonrelativistic Bethe-Heitler formula (see Heitler 1954; Bethe \& Salpeter 1957; Jackson 1975; Jung 1994a). A simple Coulomb correction to the Bethe-Heitler formula by the "Elwert-Sommerfield (ES) factor" (see Bethe \& Salpeter 1957) is well known. Recently, Gould (1990) gave a detailed discussion of the ES factor. Also, it has been shown that the ES factor can modify the electron-impact excitation cross section near thresholds (see Jung 1992). In another recent investigation by Jung (1994a), the thermal and nonthermal bremsstrahlung radiation powers including the first- and second-order Coulomb correction using the ES factor have been discussed. However, in this paper we neglect the Coulomb correction to the Bethe-Heitler formula near the cutoff spectral region and employ exclusively the Born approximation, since the investigation of the screening effects of bound electrons is the main purpose of this paper. In the following section, we will discuss the Thomas-Fermi model approximation to electron-atom bremsstrahlung radiation.

\section{THOMAS-FERMI MODEL}

As we discussed in $\$ 2.1$, our major difficulty comes from the evaluation of the Fourier transform of the atomic potential with respect to the momentum transfer $q$. For convenience, the atomic potential can also be written in the form

$$
V(\boldsymbol{r})=-\frac{Z e^{2}}{\boldsymbol{r}}+e^{2} \int d^{3} \boldsymbol{r}^{\prime} \frac{n\left(\boldsymbol{r}^{\prime}\right)}{\left|\boldsymbol{r}-\boldsymbol{r}^{\prime}\right|},
$$

where $Z$ is the nuclear charge and $n\left(r^{\prime}\right)$ is the charge distribution for the bound electrons in the target atom. Then, the Fouriertransformed potential is given by

$$
\tilde{V}(q)=-\frac{4 \pi e^{2}}{q^{2}}[Z-F(q)] .
$$

$F(q)$ is called the atomic form factor:

$$
\begin{aligned}
F(\boldsymbol{q}) & \equiv \int d^{3} \boldsymbol{r}^{\prime} n\left(\boldsymbol{r}^{\prime}\right) e^{i \boldsymbol{q} \cdot \boldsymbol{r}^{\prime}} \\
& =\frac{4 \pi}{q}\left(\frac{b}{Z^{1 / 3}}\right)^{2} \int_{0}^{\infty} d x x n(x) \sin (\bar{q} x),
\end{aligned}
$$


where $x\left(\equiv r^{\prime} Z^{1 / 3} / b\right)$ is the new dimensionless variable and $\bar{q}\left(\equiv b q / Z^{1 / 3}\right)$ is the new dimensionless momentum transfer, $b=(1 / 2)(3 \pi /$ $4)^{2 / 3} a_{0}\left(\cong 0.8853 a_{0}\right)$, and $a_{0}\left(=\hbar^{2} / m e^{2}\right)$ is the Bohr radius. If the target is a bare nucleus with charge $Z$, the factor $F(q)$ becomes zero. When the target is an atom, $F(q)$ becomes greater than zero because of the screening effects. In the Thomas-Fermi model, the number density $n(x)$ is given by (Condon $\&$ Odabasi 1980)

$$
n(x)=\frac{Z^{2}}{4 \pi b^{3}}\left[\frac{X(x)}{x}\right]^{3 / 2}
$$

where $X(x)$ is the solution of the Thomas-Fermi equation. Substituting equation (12) into equation (11b), we obtain

$$
F(\bar{q})=Z[1-\bar{q} J(\bar{q})]
$$

where

$$
J(\bar{q}) \equiv \int_{0}^{\infty} d x X(x) \sin (\bar{q} x)
$$

Therefore, the screening effects of the bound electrons can readily be obtained by $J(\bar{q})$, i.e., the solution of the Thomas-Fermi equation $X(x)$. For many purposes it is convenient to use an analytic approximation solution in exponential form (see Mott \& Massey 1965):

$$
X(x) \cong e^{-s x} \quad(s=0.66)
$$

Although the above approximation, equation (15), is not very accurate (e.g., off by $22 \%$ at $x=1$, compared with the exact expression), this simple analytic expression of the Thomas-Fermi solution is adequate for estimating the total screening effects of many electrons. It is not so difficult to show that this choice of the Thomas-Fermi solution is equivalent to the Bohr potential. Then, we have the atomic form factor:

$$
|F(Q)|^{2}=\frac{Z^{2}\left(Z^{1 / 3} \xi\right)^{4}}{\left[Q^{2}+\left(Z^{1 / 3} \xi\right)^{2}\right]^{2}},
$$

where $Q \equiv q a_{0}$ and $\xi \equiv S a_{0} / b(=0.7455)$. With this factor, the radiation cross section for electron-atom bremsstrahlung becomes

$$
\left(\frac{d \chi_{b}}{d \omega}\right)_{e^{- \text {-Alom }}}=\frac{16}{3} \frac{Z^{2} e^{2}}{c}\left(\frac{e^{2}}{m c^{2}}\right)^{2} \frac{1}{\beta^{2}} \int_{Q_{\min }}^{Q_{\max }} \frac{Q^{3} d Q}{\left(Q^{2}+\bar{\xi}^{2}\right)^{2}},
$$

where $\bar{\xi} \equiv \xi Z^{1 / 3}\left(=0.7455 Z^{1 / 3}\right)$ and other parameters are already defined in equation (8). As we mentioned before, the Coulomb correction is neglected in equation (17). The Coulomb correction in the atomic target is very different from that in the bare nucleus target because of the complex atomic potential. The correction to electron-atom bremsstrahlung will be treated elsewhere (Jung $1994 \mathrm{~b}$ ) using the effective charge method in terms of the mean radius of the total potential. A comparison is made between electron-atom and electron-nucleus bremsstrahlung cross sections in the following section.

\section{COMPARISON}

From equations (8) and (17), we can obtain the ratio of the electron-atom and electron-ion bremsstrahlung cross sections.

$$
\begin{aligned}
\frac{\left(d \sigma_{b} / d \omega\right)_{e^{-} \text {Atom }}}{\left(d \sigma_{b} / d \omega\right)_{e^{-}-Z e}} & =\frac{\left(d \chi_{b} / d \omega\right)_{e^{-} \text {-Atom }}}{\left(d \chi_{b} / d \omega\right)_{e^{--Z e}}} \\
& =\left\{\frac{1}{2} \ln \left[\frac{\bar{\xi}^{2}+\left(\sqrt{\epsilon_{0}}+\sqrt{\epsilon_{0}-\epsilon}\right)^{2}}{\bar{\xi}^{2}+\left(\sqrt{\epsilon_{0}}-\sqrt{\epsilon_{0}-\epsilon}\right)^{2}}\right]-\frac{2 \bar{\xi}^{2} \sqrt{\epsilon_{0}} \sqrt{\epsilon_{0}-\epsilon}}{\left[\bar{\xi}^{2}+\left(\sqrt{\epsilon_{0}}+\sqrt{\epsilon_{0}-\epsilon}\right)^{2}\right]\left[\bar{\xi}^{2}+\left(\sqrt{\epsilon_{0}}-\sqrt{\epsilon_{0}-\epsilon}\right)^{2}\right]}\right\} / \ln \left(\frac{\sqrt{\epsilon_{0}}+\sqrt{\epsilon_{0}-\epsilon}}{\sqrt{\epsilon_{0}}-\sqrt{\epsilon_{0}-\epsilon}}\right) .
\end{aligned}
$$

In Figure 1 the graphical comparison is given for $\mathrm{Cu}(Z=29)$ at $\epsilon_{0}=1500$. In the hard spectral region, the ratio approaches unity as $\epsilon$ approaches $\epsilon_{0}$. However, in the soft spectral region, the ratio is far from unity, so that the screening effect is significant. The screening effect is greater than $10 \%$ when the electron loses less than one-fifth of its initial energy. As $\epsilon$ increases, the screening effects become less important. Also, Figure 2 gives the graphical comparison for $\mathrm{Pb}(Z=82)$ at $\epsilon_{0}=1500$. In this case the screening effect is greater than $10 \%$ when the electron loses less than one-third of its initial energy because the screening effect is increasing with decreasing number of electrons. As we see in equation (18), the screening effect becomes relatively less important with increasing incident energy $\epsilon_{0}$. However, in the relativistic region, the screening effect increases with increasing $\epsilon_{0}$ (see Bethe \& Salpeter 1957).

\section{SUMMARY AND DISCUSSION}

In this paper we provide a simple estimation of the atomic screening effects on the electron-atom bremsstrahlung process in the nonrelativistic limit. The Born approximation is applied to describe both the initial and final states of the projectile electron. To retain the analytic procedure, we use an analytic approximate solution of the Thomas-Fermi model. Applications were made to Cu $(Z=29)$ and $\mathrm{Pb}(Z=82)$. The results show that the screening effects are significant in the soft spectral region. In both cases $(\mathrm{Cu}$ and 


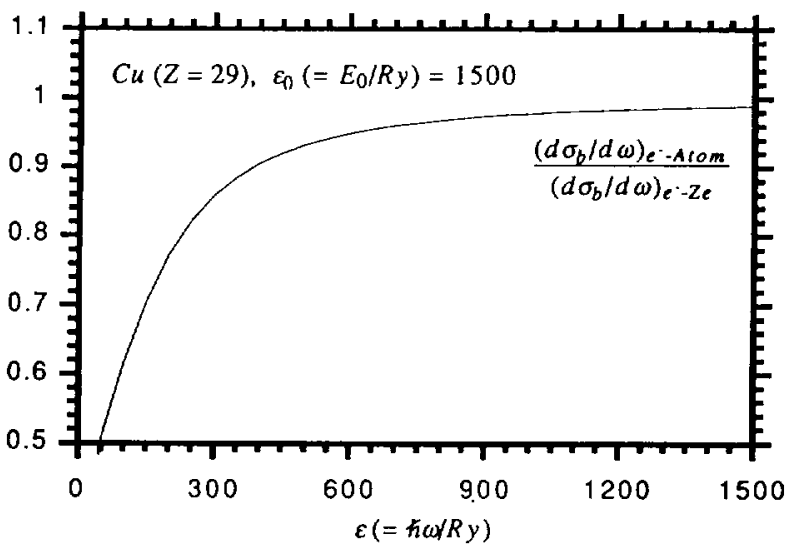

FiG. 1

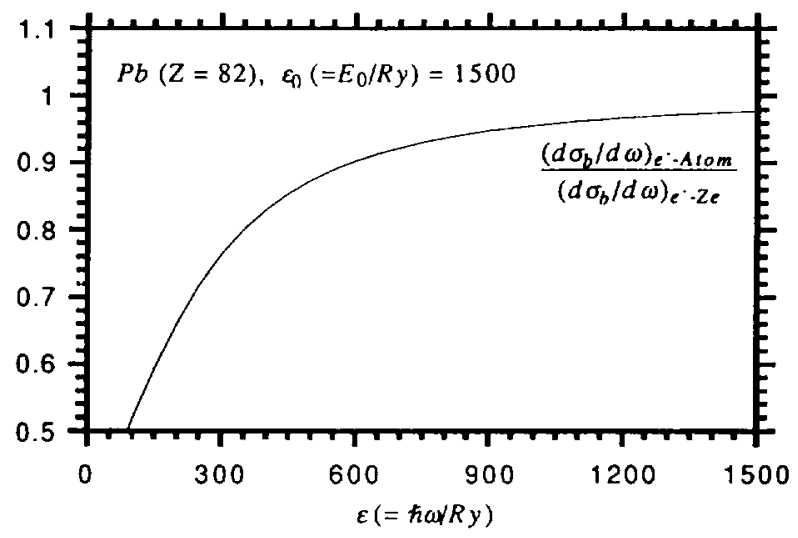

FIG. 2

FiG. 1.-Ratio (eq. [18]) of the electron-atom to electron-ion bremsstrahlung cross sections for $\mathrm{Cu}(Z=29)$ at $\epsilon_{0}=1500$

Fig. 2.-Ratio (eq. [18]) of the electron-atom to electron-ion bremsstrablung cross sections for $\mathrm{Pb}(Z=82)$ at $\epsilon_{0}=1500$

$\mathrm{Pb}$ ), when the electron loses less than one-third of its initial energy, the screening effects are more than $10 \%$. As the radiation spectrum $(\epsilon)$ increases, the screening effect becomes less important; i.e., the ratio of electron-atom to electron-ion bremsstrahlung cross sections approaches unity rapidly. Also, the screening effect is decreasing with increasing $\epsilon_{0}$. These results will be useful for obtaining correct information about the behavior of bound electrons in the target atom. Also, these results can readily be applied to a number of associated problems in atomic physics, astrophysics, and plasma physics since we obtain a simple analytic solution.

This research was supported by Hanyang University through a Faculty Research Grant (FY1993). We should also like to acknowledge the helpful comments of R. J. Gould.

\section{REFERENCES}

Bethe, H. A., \& Salpeter, E. E. 1957, Quantum Mechanics of One- and TwoElectron Atoms (New York: Academic)

Condon, E. U., \& Odabasi, H. 1980, Atomic Structure (Cambridge: Cambridge Univ. Press)

Gould, R. J. 1990, ApJ, 362, 284

Heitler, W. 1954, The Quantum Theory of Radiation (3d ed.; New York: Dover)
Jackson, J. D. 1975, Classical Electrodynamics (2d ed.; New York: Wiley)

Jung, Y.-D. 1992, ApJ, 396, 725

1994a, Phys. Plasmas, 1, 785

1994b, in preparation

Jung, Y.-D., \& Gould, R. J. 1991, Phys. Rev. A, 44, 111

Mott, N. F, \& Massey, H. S. W. 1965, The Theory of Atomic Collisions (Oxford: Oxford Univ. Press) 
- 